\title{
Techno-functional properties and in vitro bile acid-binding capacities of tamarillo (Solanum betaceum Cav.) hydrocolloids
}

\begin{abstract}
Hydrocolloids were extracted from seed mucilage and the pulp fractions from red tamarillo (Solanum betaceum Cav.) mesocarp, and characterisation of their techno-functional properties and in vitro bile acid-binding capacities was performed. The seed mucilage hydrocolloids that were extracted, using either $1 \%$ citric acid (THC) or water (THW), had a good foaming capacity (32i 36\%), whereas the pulp hydrocolloids that were extracted, using 72\% ethanol (THE) or $20 \mathrm{mM}$ HEPES buffer (THH), had no foaming capacity. The pulp hydrocolloid, however, possessed high oil-holding and water-holding capacities in the range of $3.3 \mathrm{ï} 3.6 \mathrm{~g}$ oil/g dry sample and $25 \mathrm{i} 27 \mathrm{~g}$ water/g dry sample, respectively. This enabled the pulp hydrocolloid to entrap more bile acids ( $35 \mathrm{i} 38 \%$ at a hydrocolloid concentration of $2 \%$ ) in its gelatinous network in comparison to commercial oat fibre and other hydrocolloids studied. The exceptional emulsifying properties (80ї 96\%) of both hydrocolloids suggest their potential applications as food emulsifiers and bile acid binders.
\end{abstract}

Keyword: Tamarillo; Hydrocolloids; Techno-functional properties; In vitro bile acid-binding capacities 